\title{
NUTRITION AND FUNCTIONAL FOODS \\ AT A TIME OF NATIONAL EMERGENCY: \\ LESSONS FROM A PREVIOUS NATIONAL EMERGENCY
}

\author{
Denis M. Medeiros \\ Professor of Human Nutrition \\ Kansas State University
}

Events emulating from 9-11 have made the scientific community more proactive in mitigating or preventing possible future terrorist attacks. An aspect of bioterrorism already discussed at this conference deals with maintaining a healthy food supply, and methods to combat potential pathogens and adulteration of food products aimed at harming large groups of people. Why should we be concerned with nutrition and functional foods research at a time of national emergency? We may be able to learn from the past since the science of nutrition was born out of a previous national emergency that the United States faced.

When the United States entered World War II, a large number of conscripts for the military were rejected for medical reasons. The medical basis for their maladies was often nutrition related. Keep in mind that the United States was just coming out of the Great Depression, and many parts of the nation suffered from undernutrition and exhibited signs of specific nutrient deficiency.

To help correct this problem, the U.S. Congress authorized the Food and Nutrition Board in 1940 under the direction of the National Research Council of the National Academy of Sciences. The Board was charged with making recommendations as to the levels of nutrients that should be consumed by individuals of different ages and genders to maintain health. With this charge came the Recommended Dietary Allowances, first prepared in 1941, and published in 1943. The first edition was to serve as a standard for good nutrition. The recommended intakes for the known nutrients at that time were to be used as a guide for large feeding programs and as a yardstick on which to judge adequacy of diets on a population scale. The RDAs have since been in existence and have been updated periodically every 5 to 10 years. Prior to the RDAs, nutrition research had been practiced in various labs throughout the country and elsewhere in the world, but in a piecemeal fashion. Physicians, chemists and physiologists conducted much of the work. Nutrition as a science was in its early evolution when World War II erupted. That national emergency galvanized the field into becoming a modern discipline.

Given the history of nutrition research, it is not surprising that research today continues to focus on deficiencies of essential macronutrients and micronutrients, their roles in living organisms, and the mechanism of actions. Research in the twentieth century has led to the identification and 
characterization of essential nutrients and has provided the basis for dietary guidelines for optimal health throughout the life span. Furthermore, research findings have firmly established the importance of proper nutrition for maintaining good health, and also preventing the development of various degenerative diseases associated with affluence. We have seen a substantial rise in overnutrition, life-style changes, and also the incidence and prevalence of chronic diseases such as coronary heart disease, diabetes, and various cancers associated with affluence. Dietary intervention, along with life style modification, has become a primary strategy for preventing and treating such diseases. Genetics has an important role in the development of such diseases, but a focal point of nutrition research in recent years has been the impacts of nutrients and other bioactive components on health via their interactions with specific genes.

The RDAs that were developed out of a national emergency have since had widespread application, among the most important being the subsidized federal programs like school lunch and Medicare-funded programs. The Food and Drug Administration uses the RDA's as a guide for food labeling. Scientists have used them as a way to judge the adequacy of diets for various parts of the population and to identify high-risk groups. Since a change in a value of one nutrient, up or down, can mean a change in how much of a certain food type must be present in a feeding program to receive federal dollars, the political aspects of the RDAs may be contentious.

In the last decade, much attention has been directed toward the health benefits of such food components as fibers, carotenoids, tocopherols, isoflavones, polyphenols, terpenes, conjugated linoleic acids, marine oils, and other organic compounds such as protease inhibitors and saponins found in grains, oil seeds, and vegetables. Epidemiological studies have shown that the consumption of foods rich in these compounds of plant and animal origin is associated with a reduced incidence of coronary heart disease, cancer, osteoporosis, or other degenerative diseases. Only recently, researchers have begun to uncover the biological and physiological effects of certain bioactive components of foods, which sharply increased our awareness of their potential importance in health maintenance and disease prevention. Some important findings include: the anticarcinogenic potential of conjugated linoleic acids, carotenoids, isoflavones, saponins, and inositol hexaphosphate; inhibition of bone resorption by phytoestrogens in women; the cholesterol-lowering effect of dietary polyphenols; stimulatory action of a soy protein component(s) on thyroxine secretion; antiatherogenic and anticarcinogenic effects of conjugated linoleic acids, n-3 fatty acids, and tocopherols; and preventive effects of Lcarnitine and natural and synthetic antioxidants on neurodegeneration and cognitive dysfunction.

Clearly, nutrition and food research for the twenty-first century will place great emphasis on bioactive functional components in grains and oil seeds as well as animal and marine products. The search for new bioactive compounds 
will continue with renewed vigor. In recent years, funding from the federal agencies and industry for research in these areas has increased substantially and is expected to increase in response to the ever-increasing public demand for information on dietary supplements and the health effects of plant- and animalbased functional/designer foods and nutraceuticals. This new direction will have a significant impact on public health. Information generated from the research will undoubtedly influence future strategies for nutritional intervention in health and disease. It is anticipated that those functional components of foods proven to be effective in prevention and treatment of diseases will be commercially manufactured and marketed as nutraceuticals and functional ingredients of processed foods. However, given the shifting national priorities of post 9-11, it is not clear whether other issues will take priority over this evolving science. Similarly, great strides have been made with the human genome project. Will funding for applications coming from this work be curtailed and replaced by research on bioterrorism? In the case of functional foods, many of these new products have been modified in some form, some genetically altered. This poses an additional point of entry from which harmful components to the food supply could be added.

These issues are not to be taken lightly for states such as Kansas, Nebraska, lowa and Missouri, where there is a large agricultural base on which to capitalize. Investment in nutrition has been upward and steady. The interest in these food products has intensified among the food industry over the last 10 years. In 1992, the market for functional foods was $\$ 5.4$ billion, and by 2001 the market was expected to be over $\$ 18$ billion. It is not clear which food companies will continue to invest in this emerging area of interest, who will become the market leaders, and what the target audience will be for many of their products. Nevertheless, it is clear that the markets for functional foods are large and growing, ranging from specialty niches to mass-market opportunities. For agricultural states, the challenge is sustaining momentum for nutrition research while other needs outweigh the priorities established prior to our recent national emergency. On the other hand, we could experience long-term economic benefits as we shift research dollars to combating bioterrorism. Clearly, we have observed the many benefits that the space program has given modern medicine from its spin-off technologies. Another concern is the bandwagon effect everyone jumps to try and capture new short-lived research dollars, and the infrastructure that was used to create the research support becomes obsolete in a short amount of time. Clearly research administrators and researchers must keep both the short term and long term perspective in making adjustments to their grantsmanship strategies and institutional investments. 
References

Position of the American Dietetics Association: Functional Foods. (1999). Journal of the American Dietetic Association, 99, 1278-1285.

Recommended Dietary Allowances. (1989) (10 ${ }^{\text {th }}$ ed.). Washington, D.C.: National Research Council.

Wildman, R.E.C. (2001). Handbook of Nutraceuticals and Functional Foods. Boca Raton, FL: CRC Press.

Wildman, R.E.C. (2000). Advanced Human Nutrition. Boca Raton, FL: CRC Press. 